\title{
Radiation dose escalation for locally advanced unresectable intrahepatic and extrahepatic cholangiocarcinoma
}

\author{
Santiago Avila, Danyal A. Smani, Eugene J. Koay \\ Department of Radiation Oncology, The University of Texas MD Anderson Cancer Center, Houston, TX, USA \\ Contributions: (I) Conception and design: All authors; (II) Administrative support: EJ Koay; (III) Provision of study materials or patients: All authors; \\ (IV) Collection and assembly of data: All authors; (V) Data analysis and interpretation: All authors; (VI) Manuscript writing: All authors; (VII) Final \\ approval of manuscript: All authors. \\ Correspondence to: Eugene J. Koay, MD, PhD. Radiation Oncology Department, The University of Texas MD Anderson Cancer Center, 1515 \\ Holcombe Blvd Unit 1422, Houston, Texas 77030, USA. Email: ekoay@mdanderson.org.
}

\begin{abstract}
Intrahepatic cholangiocarcinoma (IHCC) and extrahepatic cholangiocarcinoma (EHCC) remain challenging diseases to treat. The majority of patients present with advanced disease, and the tumors often cause life-threatening biliary obstruction and vascular compromise of the liver. Local control (LC) of these tumors has the potential to prolong life for patients. While escalated-dose radiation therapy (EDRT) has been demonstrated to be an effective, safe option to achieve LC of IHCC, data for EHCC suggest that EDRT with current techniques has limitations, often due to dose-limiting bowel structures in close proximity to the extrahepatic biliary system. Here we review the results of EDRT for IHCC and EHCC and point to potential directions to combine radiotherapy with novel agents. The molecular characterization of cholangiocarcinoma has particularly opened new avenues for clinical investigations of targeted therapies with EDRT and may point to ways to achieve both systemic and LC benefits for patients.
\end{abstract}

Keywords: Cholangiocarcinoma; radiation; targeted therapy

Submitted Aug 31, 2019. Accepted for publication Dec 05, 2019.

doi: $10.21037 /$ cco.2019.12.05

View this article at: http://dx.doi.org/10.21037/cco.2019.12.05

\section{Introduction}

\section{Overview}

Cholangiocarcinoma is a lethal disease of the biliary tract (1). Depending on the anatomical location (Figure 1), tumors are either classified as intrahepatic or extrahepatic. These tumors present numerous challenges for patients and physicians: advanced disease presentation for the majority of patients, recurrence after surgery, therapeutic resistance, and medical complications caused by the tumors. For both intrahepatic cholangiocarcinomas (IHCC) and extrahepatic cholangiocarcinomas (EHCC), respectively, medical complications such as biliary obstruction and vascular compromise of the liver are often life-threatening, emphasizing that LC of these tumors is paramount for many patients. The management of these different biliary tract cancers to achieve LC differs in important ways.

In patients with early stage, resectable cancers, LC is often adequately achieved with surgery. However, for advanced stage (locally advanced and metastatic) presentations, surgery is not usually a viable option. Most patients will only receive palliative chemotherapy when they have advanced disease, but emerging evidence suggests that prolongation of life for patients with unresectable locally advanced IHCC can be achieved with escalated dose radiation therapy (EDRT) (2,3). Application of EDRT to EHCC (4), on the other hand, has not been associated with prolonged survival largely due to limitations of giving higher radiation doses near bowel and stomach. Here, we review the recent data with EDRT for IHCC and EHCC, and point to areas of future investigation in an era of molecular characterization and personalized medicine for 


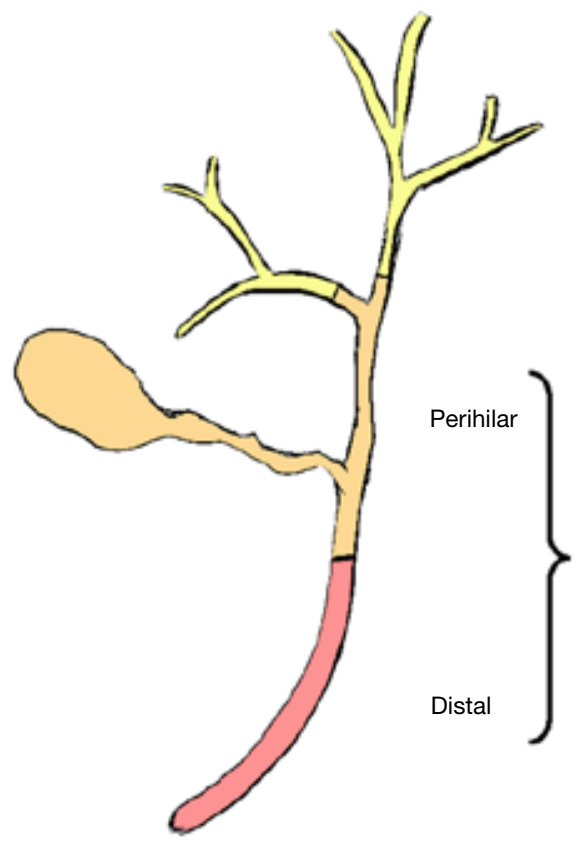

Intrahepatic

Primary causes of death:

- Biliary complications (44\%)

- Vascular occlusion (28\%)

- Parenchymal liver failure (6\%)

- Combination of above (11\%)

- Extrahepatic disease burden (11\%)

\section{Extrahepatic}

Primary causes of death:

- Biliary complications (14\%)

- Vascular occlusion (5\%)

- Cholangitis (16\%)

- Parenchymal liver failure $(7 \%)$

- Combination of above (21\%)

- Extrahepatic disease burden (27.3\%)

- Organ failure (11.4\%)

Figure 1 Anatomical and clinical differences between intrahepatic and extrahepatic cholangiocarcinoma. Though higher percentages of extrahepatic cholangiocarcinoma (EHCC) patients die from extrahepatic disease burden, the high percentage of patients dying from local complications point towards the importance of local control.

cholangiocarcinoma (5).

\section{History of radiation therapy in the treatment of cholangiocarcinoma}

Historically, the utilization of radiation therapy for liver lesions has been limited by the risk for radiation-induced liver disease (RILD) (6). However, the use of focal high doses of radiation was shown to be safe in a series of seminal studies in the 1990s and 2000s $(7,8)$. Since those studies, the role of radiation therapy in the management of liver cancers has been expanding. The advent of technological advances such as intensity-modulated RT (IMRT), respiratory management methods, and improved image guidance have enabled potentially ablative doses for the treatment of cholangiocarcinoma (2).

\section{Importance of $L C$}

Several studies have shown that local progression in IHCC and EHCC often leads to liver failure and subsequent death (2-4). Tao et al. demonstrated that a majority of patients with unresectable IHCC die from tumor-related liver failure due to vascular compromise or biliary obstruction (3).
A follow-up study that included surgical cases demonstrated that, within a cohort of patients with IHCC and for which cause of death was known, 54\% died from tumor progression leading to tumor-related liver failure (9). LC is similarly important in EHCC. While a higher proportion of patients with EHCC die of extrahepatic disease burden or other organ failure compared to IHCC, local tumor-related complications remain an important contributor to patient death (4).

\section{Liver-directed treatment options for IHCC and EHCC}

We previously reviewed the current management options of unresectable IHCC (10) and more generally discussed the technological advancements that enable ablative radiation doses for large liver lesions (Figure 2) (2). Possible alternatives to external beam radiation therapy include, radioemolization with Yttrium-90, radiofrequency ablation, and transarterial chemoembolization. The rationale to use a given liver-directed therapy is largely based on physician experience at a given hospital and availability of the technology at a given center. Level 1 evidence does not exist to show that one therapy is better than another. When all of these options are available at an institution, multi- 

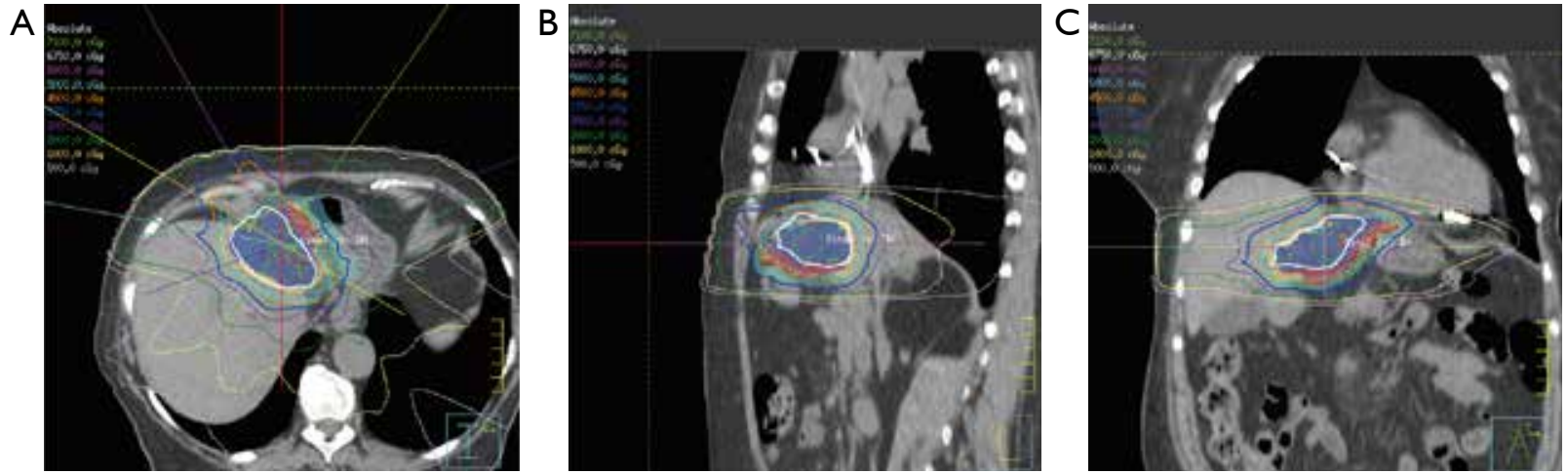

Figure 2 Radiation treatment plans for a patient treated with dose-escalated RT for intrahepatic cholangiocarcinoma. The plans show dose distribution lines in the axial plane (left), sagittal plane (middle), and coronal plane (right).

disciplinary management will help ensure an individualized approach for a patient, as clinical, biological, and anatomical considerations can be factored into the decision-making process.

\section{Dose-escalation for cholangiocarcinoma}

\section{IHCC}

Surgery is widely regarded as the only potentially curative option for patients with IHCC, but few patients with IHCC are candidates for radical resection $(\sim 10-15 \%)(11)$. Instead, patients with inoperable disease receive cisplatin plus gemcitabine, which showed improved overall survival (OS) compared to gemcitabine alone (12).

Interest in using radiation therapy for IHCC dates back decades, but early studies were constrained to low doses and conventional fractionation due to normal tissue dose constraints. In the past 15 years, advances in radiation delivery, including intensity modulated radiation therapy (IMRT), proton therapy respiratory gating and CTimage guidance, contributed to the ability to safely deliver escalated doses of radiation (EDRT). These advances were utilized in Japan for hepatocellular carcinoma (HCC) where hypofractionated proton therapy to escalated doses achieved 5 -year LC (90\%) and OS (50\%) rates similar to those after surgical resection for large tumors (10). A retrospective study of 79 patients treated with conventional and EDRT therapy for IHCC suggested a dose-response correlation. This study found that biological equivalent dose (BED) as a continuous variable significantly associated with LC and OS. Patients who received a BED of $>80.5$ Gy had a $73 \%$ 3 -year OS compared to $38 \%$ for those receiving lower doses
$(\mathrm{P}=0.017)$. The higher radiation doses were linked with a lower rate of tumor-related liver failure, indicating that the higher BED treatments were a major contributor to the substantial prolongation of life that was observed (3).

A follow-up study investigated outcomes of 362 historical IHCC patients from 1997 to 2015 treated with either definitive radiation, resection, or chemotherapy (9). Clinicopathological characteristics, cause of death, diseasespecific survival (DSS), and intrahepatic progressionfree survival (IPFS) were compared between groups and within groups by era. This study found no significant improvement over time in IPFS or DSS for patients treated with definitive chemotherapy. Meanwhile IPFS improved from $50 \%$ to $75 \%$ and $48 \%$ to $64 \%$ for resection and radiation respectively. DSS too improved from $58 \%$ to $67 \%$ and $12 \%$ to $37 \%$ for resection and radiation respectively. Additionally, both forms of local therapy were associated with fewer liver related deaths for patients with IHCC (30\% resection, $41 \%$ radiation, $72 \%$ chemotherapy). The improvement in IPFS and DSS for radiation was associated with EDRT. The focal delivery of higher radiation doses was hypothesized to confer a survival benefit similar to surgery because both therapies were associated with a lower likelihood of tumor-related liver failure, unlike systemic therapy.

Prospective evaluation of EDRT delivered with protons was shown in a study of 44 patients with HCC, 37 patients with IHCC, and 2 patients with mixed HCC/IHCC. Patients received 15 fractions of radiation to a maximum of 67.5 Gy equivalent. The 2-year LC for IHCC was $94.1 \%$ and the 2-year OS for IHCC was $46.5 \%$ (13). Encouraging results were also noted for HCC. This study led to two phase 3 randomized trials, NRG GI001 for IHCC 

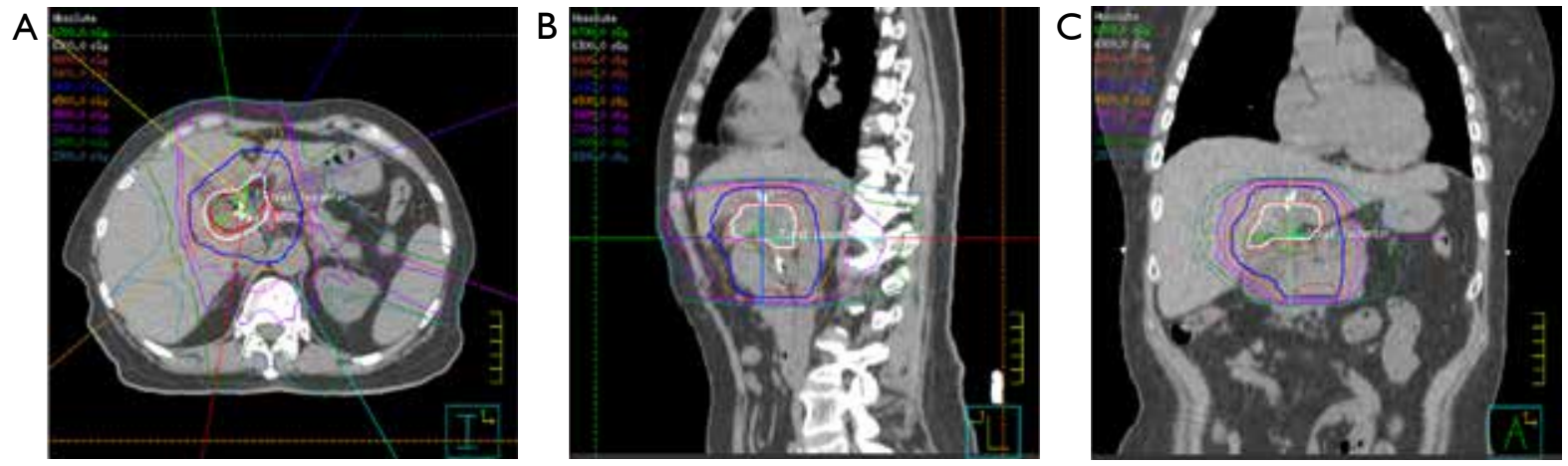

Figure 3 Radiation treatment plans for a patient treated with dose-escalated RT for extrahepatic cholangiocarcinoma. The plans show dose distribution lines in the axial plane (left), sagittal plane (middle), and coronal plane (right).

(hypofractionated radiation $v s$. observation after initial chemotherapy, NCT02200042) and NRG GI003 for HCC (protons vs. photons, NCT03186898). The NRG GI001 was terminated due to lack of accrual, and the NRG GI003 study is ongoing.

An analysis of the National Cancer Database revealed that between the years 2004-2013, 401 out of 4,374 patients (9.2\%) with IHCC were treated with radiation therapy (14). Patients who received radiation had longer median survival times, and receipt of radiation therapy was an independent predictor of prolonged survival in this large study of a contemporary cancer database. Notably, patients who lived in certain geographic areas and who received treatment at community centers were less likely to receive radiation. These results further emphasize the importance of LC in IHCC, the emerging role of radiation therapy in managing this disease, and the need to better educate physicians about how to deliver radiation at high doses.

\section{EHCC}

The results of EDRT in EHCC have not been as clearly favorable as those for IHCC. In 1990, a multi-center, retrospective study of patients with EHCC reported an improved median survival in patients receiving $>40.0 \mathrm{~Gy}$ compared to those receiving $\leq 40.0$ Gy (15). A retrospective analysis of 52 patients with unresectable EHCC treated between 1957 and 2000 at The University of Texas MD Anderson Cancer Center shed light on the limitations of conventional dosing in preventing local progression (16). Though the study was limited in its statistical power by its small patient number, it did suggest a possible association between increasing radiation dose and improved LC.
However, in a recent study, we retrospectively analyzed a consecutive cohort of 80 patients treated with RT for unresectable EHCC between 2001 and 2015 (4). RT was given to doses of 30-75 Gy (median 50.4) for a biological equivalent dose (BED) of 36-98 Gy (median 59.5). The cohort was split into an escalated-dose RT (EDRT) group (>50.4 Gy in 28 fractions, BED >59.5) and a conventional dose group (BED $\leq 59.5)$. The EDRT group did not demonstrate improved OS or freedom from local progression (FFLP). Further, EDRT was associated with worse freedom from distant progression and was also associated with the onset of grade 3 or higher lymphopenia, which has been shown to portend poor prognosis in other disease sites (17). All other toxicity rates were comparable. While dose-escalation has shown promising results in IHCC, these results suggest that higher doses do not provide the same LC and OS benefits in EHCC. The proximity of EHCC tumors to bowel limit the ability to completely cover the tumor with higher doses of radiation (Figure 3).

The field still faces the challenge of achieving better LC for EHCC. Indeed, tumor-related complications are a significant driver of morbidity and mortality in patients with EHCC (4). There is a need to intensify treatment for EHCC, and new systemic agents, in combination with radiation, may provide a solution.

\section{Combining targeted therapy and radiation therapy}

As EDRT for IHCC has shown promising LC and patients live longer, secondary patterns of regional and distant failure outside of the radiation fields have become apparent. Our 
group presented an update to our experience with EDRT for IHCC, examining 63 patients with IHCC treated from 2010 to 2016 with hypofractionated RT (Abu-Gheida, Koay et al., American Association for Clinical Oncology 2019 Annual meeting abstract). This update showed good LC and OS rates (local-progression-free-survival of $67 \%$ and 2 -year OS of $71 \%$ ) and low toxicity rates. However, intrahepatic-distant-metastasis-free-survival (intrahepatic metastasis outside of the radiation field) and extrahepaticdistant-metastasis-free-survival were both $40 \%$, indicating that systemic control remains a major challenge.

Thus, there is strong rationale to combine systemic therapy with radiation therapy to achieve both distant and LC. In particular, the combination of targeted agents with EDRT represents an emerging opportunity for clinical research. Several molecular targets have been identified in recent years for cholangiocarcinoma, including tumors with fibroblast growth factor receptor genetic alterations (FGFR GAs), isocitrate dehydrogenase-1 (IDH1) mutations, and high microsatellite instability.

\section{FGFR}

FGFR GAs appear to represent a unique subtype of IHCC, occurring in $10 \%$ to $16 \%$ of IHCC cases. Patients with these genetic alterations appear to have a more indolent disease course and are generally younger than the patients without FGFR GAs (18). Javle and associates studied an orally bioavailable, selective pan-FGFR kinase inhibitor BGJ398 (infigratinib) in patients with FGFR2 fusions or other FGFR alterations whose disease had progressed while receiving prior therapy. The overall response rate was $14.8 \%$ and the disease control rate was $75.4 \%$. The median progression free survival was 5.8 months (19). An ongoing phase 3 study for advanced cholangiocarcinoma with FGFR GAs is testing this drug in the frontline setting, randomizing the patients to infigratinib versus gemcitabine/ cisplatin (NCT03773302). Other FGFR inhibitors, including TAS-120, JNJ-42756493, AZD4547 are similarly being tested in clinical trials.

The mechanism of action for FGFR GAs has been a topic of great interest due to its role in cholangiocarcinoma as well as multiple other cancer types. Upon ligand binding, the transmembrane protein dimerizes to trigger multiple downstream signalling pathways, including mitogen activated protein kinase, signal transducer and activator of transcription (STAT), and the phosphoinositide-3kinase (PI3K-Akt) pathway (20). Through these and other pathways, FGFR GAs are thought to contribute to oncogenesis through cell growth and survival, angiogenesis, and resistance to therapy. Preclinical studies have shown radiosensitization with FGFR inhibition likely is dependent on the specific genetic alteration. Ahmed et al demonstrated that FGFR 4 contributes to radioresistance in colorectal cancer through reduction of RAD51 levels, thereby making HT29 colorectal cell lines resistant to radiation therapy. Silencing of FGFR4 radiosensitized the HT29 cells by attenuation of double strand break repair by homologous recombination (21). Verstraete $e$ al. investigated the ability of FGFR inhibition with JNJ-42756493 to radiosensitize colorectal xenograft models with wild type FGFR and FGFR2 amplification, but did not observe a radiosensitizing effect (22). There is no available data on FGFR inhibition and radiation therapy for IHCC, and it may be that only specific subsets of FGFR GAs benefit from a combination of FGFR inhibition and radiation. More basic and clinical research in this area is needed.

\section{IDH1}

IDH1 is mutated in about $25 \%$ of cholangiocarcinomas and has been tested in patients with a phase 1 dose-escalation study with AG-120. In a cohort of patients with mutated IDH1 cholangiocarcinoma, the partial response rate was $5 \%$ (4 out of 73 patients). The median PFS was 3.8 months and median OS was 13.8 months (23). Preliminary results of an ongoing phase 3 trial of AG-120 versus placebo in mutated IDH1 cholangiocarcinoma has been presented at ASCO (Lowery 2017 ASCO Annual Meeting) and has the primary endpoint of PFS.

Mutations in IDH1/2 occur in a hotspot of the catalytically active site of action for these enzymes, resulting in production of D-2-hydroxyglutarate (D-2HG). D-2HG competitively binds alpha-ketoglutarate, causing alterations in multiple downstream cellular processes like cell metabolism, DNA repair, redox states, and epigenetic regulation (24). Patients with IDH1/2 mutations have been shown to have improved responses to radiation in glioma $(25,26)$. In preclinical studies, the silencing of wild type IDH1 has shown sensitization of cancer cells to chemotherapy and radiation, indicating that the targeting of wild type IDH1/2 cancers with pharmacologic inhibition may confer the same therapy responses seen in mutant IDH1/2 cancers. Indeed, this concept has been shown in preclinical studies $(27,28)$. In the mutant IDH $1 / 2$ population, targeted therapies may also be rationally 
combined with radiation or chemotherapy to further sensitize the cancers to treatment. For example, mutated IDH1/2 cancers can have a "BRCAness" phenotype due to impaired homologous recombination DNA repair, that is partly attributed to inhibition of the alpha-ketoglutaratedependent dioxygenases [DNA damage response proteins lysine-specific demethylase 4A/B (KDM4A/B)], which induces the DNA repair defect. This has led researchers to investigate the use of poly ADP ribose polymerase (PARP) inhibitors in IDH 1/2-mutated cancers. Similarly, studies have indicated that BCL2 and NAMPT may also be good targets in this subtype of cancer, including cholangiocarcinoma (24). More clinical investigation is warranted to determine how radiation can be combined with targeted agents for IDH 1/2, both in mutated and nonmutated situations.

\section{MSI high}

Around $1 \%$ of cholangiocarcinomas are thought to have high level microsatellite instability $(29,30)$. At present, the data for checkpoint blockade in cholangiocarcinoma are limited. The initial report of the KEYNOTE-028 trial for PDL1-positive biliary tract cancers showed partial response in 4 of 24 patients $(17 \%)$ and an additional 4 patients $(17 \%)$ with stable disease (31). This led to a successor trial, KEYNOTE-158, which included 104 patients with biliary tract cancers. The objective response rate for the patients with biliary tract cancers was only $5.8 \%$ in this study and 17 patients $(16 \%)$ had stable disease. Responses have also been documented in patients with mismatch repair deficient cholangiocarcinoma (32), with all 4 cholangiocarcinoma patients in a basket study of 86 with MMR-deficient tumors showing stable disease $(n=3)$ or complete response $(n=1)$. Thus, checkpoint blockade may benefit a subset of patients with cholangiocarcinoma.

Several recently reported trials have described abscopal responses in patients who have received radiation therapy and immune checkpoint blockade. In unirradiated tumors on these trials, the abscopal response rates ranged from $10-27 \%$ with another $13-23 \%$ having stable disease (33-35). In these trials, the responses in unirradiated tumors correlated with an increase in the peripheral CD8+ T cells, peripheral immune cells, antibodies, and tumor-infiltrating cytotoxic T cells. Further research will be needed to maximize the anti-tumor effects of the immune system for cholangiocarcinoma and understand what role radiation may play in achieving this.

\section{Future directions and summary}

The goal of achieving LC of IHCC and EHCC stems from the fact that most patients with these diseases experience medical complications related to the primary tumor that can be life threatening, including biliary obstruction and vascular compromise. Controlling these tumors with liverdirected therapies like surgical resection, ablation, and radiation therapy can prolong life for patients. Giving EDRT using modern technologies has shown promise for IHCC, but the data for EHCC do not indicate the same benefit. These different results with EDRT in IHCC and EHCC are likely due in part to limitations of covering all EHCC tumors with high doses of radiation, owing to nearby bowel structures that are sensitive to high doses of radiation. Improved outcomes for patients in terms of LC will thus require new strategies, and targeted agents, particularly for FGFR gene aberrations, IDH1/2 mutations, and MSI-high, offer multiple avenues for clinical research with radiation therapy.

\section{Acknowledgments}

We gratefully acknowledge support from the Andrew Sabin Family Fellowship, the Sheikh Ahmed Center for Pancreatic Cancer Research, institutional funds from The University of Texas MD Anderson Cancer Center, equipment support by GE Healthcare and the Center of Advanced Biomedical Imaging, Philips Healthcare, and Cancer Center Support (Core) Grant CA016672 from the National Cancer Institute to MD Anderson. Dr. Eugene Koay was supported by NIH (U54CA210181-01, 1P50CA22170701A1, 1R01CA218004-01A1, 1R01CA221971-01A1, 1U01CA200468-01, and 1U01CA196403-01), Stand Up to Cancer, Project Purple, and the Khalifa Foundation.

\section{Footnote}

Conflicts of Interest: The authors have no conflicts of interest to declare.

Ethical Statement: The authors are accountable for all aspects of the work in ensuring that questions related to the accuracy or integrity of any part of the work are appropriately investigated and resolved.

\section{References}

1. Blechacz B. Cholangiocarcinoma: Current Knowledge and 
New Developments. Gut Liver 2017;11:13-26.

2. Crane CH, Koay EJ. Solutions that enable ablative radiotherapy for large liver tumors: Fractionated dose painting, simultaneous integrated protection, motion management, and computed tomography image guidance. Cancer 2016;122:1974-86.

3. Tao R, Krishnan S, Bhosale PR, et al. Ablative Radiotherapy Doses Lead to a Substantial Prolongation of Survival in Patients With Inoperable Intrahepatic Cholangiocarcinoma: A Retrospective Dose Response Analysis. J Clin Oncol 2016;34:219-26.

4. Elganainy D, Holliday EB, Taniguchi CM, et al. Dose escalation of radiotherapy in unresectable extrahepatic cholangiocarcinoma. Cancer Med 2018;7:4880-92.

5. Andersen JB, Spee B, Blechacz BR, et al. Genomic and genetic characterization of cholangiocarcinoma identifies therapeutic targets for tyrosine kinase inhibitors. Gastroenterology 2012;142:1021-31.e15.

6. Koay EJ, Owen D, Das P. Radiation-Induced Liver Disease and Modern Radiotherapy. Semin Radiat Oncol 2018;28:321-31.

7. Dawson LA, McGinn CJ, Normolle D, et al. Escalated focal liver radiation and concurrent hepatic artery fluorodeoxyuridine for unresectable intrahepatic malignancies. J Clin Oncol 2000;18:2210-8.

8. Lawrence TS, Ten Haken RK, Kessler ML, et al. The use of 3-D dose volume analysis to predict radiation hepatitis. Int J Radiat Oncol Biol Phys 1992;23:781-8.

9. Yamashita S, Koay EJ, Passot G, et al. Local therapy reduces the risk of liver failure and improves survival in patients with intrahepatic cholangiocarcinoma: A comprehensive analysis of 362 consecutive patients. Cancer 2017;123:1354-62.

10. Koay EJ, Odisio BC, Javle M, et al. Management of unresectable intrahepatic cholangiocarcinoma: how do we decide among the various liver-directed treatments? Hepatobiliary Surg Nutr 2017;6:105-16.

11. Wang K, Zhang H, Xia Y, et al. Surgical options for intrahepatic cholangiocarcinoma. Hepatobiliary Surg Nutr 2017;6:79-90.

12. Valle J, Wasan H, Palmer DH, et al. Cisplatin plus gemcitabine versus gemcitabine for biliary tract cancer. $\mathrm{N}$ Engl J Med 2010;362:1273-81.

13. Hong TS, Wo JY, Yeap BY, et al. Multi-Institutional Phase II Study of High-Dose Hypofractionated Proton Beam Therapy in Patients With Localized, Unresectable Hepatocellular Carcinoma and Intrahepatic Cholangiocarcinoma. J Clin Oncol 2016;34:460-8.
14. Kolarich AR, Shah JL, George TJ Jr, et al. Nonsurgical management of patients with intrahepatic cholangiocarcinoma in the United States, 2004-2015: an NCDB analysis. J Gastrointest Oncol 2018;9:536-45.

15. González González D, Gerard JP, Maners AW, et al. Results of radiation therapy in carcinoma of the proximal bile duct (Klatskin tumor). Semin Liver Dis 1990;10:131-41.

16. Crane CH, Macdonald KO, Vauthey JN, et al. Limitations of conventional doses of chemoradiation for unresectable biliary cancer. Int J Radiat Oncol Biol Phys 2002;53:969-74.

17. Grossman SA, Ellsworth S, Campian J, et al. Survival in Patients With Severe Lymphopenia Following Treatment With Radiation and Chemotherapy for Newly Diagnosed Solid Tumors. J Natl Compr Canc Netw 2015;13:1225-31.

18. Jain A, Borad MJ, Kelley RK, et al. Cholangiocarcinoma with FGFR genetic aberrations: A unique clinical phenotype. JCO Precis Oncol 2018. doi: 10.1200/ PO. 17.00080

19. Javle M, Lowery M, Shroff RT, et al. Phase II Study of BGJ398 in Patients With FGFR-Altered Advanced Cholangiocarcinoma. J Clin Oncol 2018;36:276-82.

20. Chae YK, Ranganath K, Hammerman PS, et al. Inhibition of the fibroblast growth factor receptor (FGFR) pathway: the current landscape and barriers to clinical application. Oncotarget 2017;8:16052-74.

21. Ahmed MA, Selzer E, Dorr W, et al. Fibroblast growth factor receptor 4 induced resistance to radiation therapy in colorectal cancer. Oncotarget 2016;7:69976-90.

22. Verstraete M, Debucquoy A, Gonnissen A, et al. In vitro and in vivo evaluation of the radiosensitizing effect of a selective FGFR inhibitor (JNJ-42756493) for rectal cancer. BMC Cancer 2015;15:946.

23. Lowery MA, Burris HA 3rd, Janku F, et al. Safety and activity of ivosidenib in patients with IDH1-mutant advanced cholangiocarcinoma: a phase 1 study. Lancet Gastroenterol Hepatol 2019;4:711-20.

24. Molenaar RJ, Maciejewski JP, Wilmink JW, et al. Wildtype and mutated IDH1/2 enzymes and therapy responses. Oncogene 2018;37:1949-60.

25. Buckner JC, Shaw EG, Pugh SL, et al. Radiation plus Procarbazine, CCNU, and Vincristine in Low-Grade Glioma. N Engl J Med 2016;374:1344-55.

26. Cairncross JG, Wang M, Jenkins RB, et al. Benefit from procarbazine, lomustine, and vincristine in oligodendroglial tumors is associated with mutation of IDH. J Clin Oncol 2014;32:783-90. 
27. Calvert AE, Chalastanis A, Wu Y, et al. CancerAssociated IDH1 Promotes Growth and Resistance to Targeted Therapies in the Absence of Mutation. Cell Rep 2017;19:1858-73.

28. Zarei M, Lal S, Parker SJ, et al. Posttranscriptional Upregulation of IDH1 by HuR Establishes a Powerful Survival Phenotype in Pancreatic Cancer Cells. Cancer Res 2017;77:4460-71.

29. Goeppert B, Roessler S, Renner M, et al. Mismatch repair deficiency is a rare but putative therapeutically relevant finding in non-liver fluke associated cholangiocarcinoma. Br J Cancer 2019;120:109-14.

30. Winkelmann R, Schneider M, Hartmann S, et al. Microsatellite Instability Occurs Rarely in Patients with Cholangiocarcinoma: A Retrospective Study from a German Tertiary Care Hospital. Int J Mol Sci 2018;19. doi: 10.3390/ijms19051421.

31. Bang YJ, Doi T, De Braud F, et al. Safety and Efficacy of

Cite this article as: Avila S, Smani DA, Koay EJ. Radiation dose escalation for locally advanced unresectable intrahepatic and extrahepatic cholangiocarcinoma. Chin Clin Oncol 2020;9(1):10. doi: 10.21037/cco.2019.12.05
Pembrolizumab in Patients with Advanced Biliary Tract Cancer: Interim Results of KEYNOTE-028. Euro J Cancer 2015;51:S112.

32. Le DT, Durham JN, Smith KN, et al. Mismatch repair deficiency predicts response of solid tumors to PD-1 blockade. Science 2017;357:409-13.

33. Hiniker SM, Reddy SA, Maecker HT, et al. A Prospective Clinical Trial Combining Radiation Therapy With Systemic Immunotherapy in Metastatic Melanoma. Int J Radiat Oncol Biol Phys 2016;96:578-88.

34. Twyman-Saint Victor C, Rech AJ, Maity A, et al. Radiation and dual checkpoint blockade activate non-redundant immune mechanisms in cancer. Nature 2015;520:373-7.

35. Tang C, Welsh JW, de Groot P, et al. Ipilimumab with Stereotactic Ablative Radiation Therapy: Phase I Results and Immunologic Correlates from Peripheral T Cells. Clin Cancer Res 2017;23:1388-96. 